\title{
B and $\Lambda_{b}$ lifetimes at ATLAS and CMS
}

\author{
C. Schiavi* \\ On behalf of the ATLAS and CMS Collaborations \\ Dipartimento di Fisica dell'Università di Genova and I.N.F.N. Genova \\ E-mail: Carlo.Schiaviecern.ch
}

\begin{abstract}
The status of some beauty lifetime measurements performed by the ATLAS and CMS experiments at the LHC is reviewed, focusing on $B$ hadrons and the $\Lambda_{b}^{0}$ baryon. The lifetimes of $B_{s}^{0}$ and $B_{d}^{0}$ mesons are determined from their decays $B_{d}^{0} \rightarrow J / \psi K^{*}$ and $B_{s}^{0} \rightarrow J / \psi \phi$. For the $\Lambda_{b}^{0}$ baryon, the lifetime and its ratio to $B_{d}^{0}$ lifetime is extracted studying the decay $\Lambda_{b}^{0} \rightarrow J / \psi \Lambda^{0}$. In all these analyses, the $J / \psi$ is reconstructed in the decay $J / \psi \rightarrow \mu^{+} \mu^{-}$. Results are obtained using $p p$ collision data at a center-of-mass energy of $7 \mathrm{TeV}$.
\end{abstract}

14th International Conference on B-Physics at Hadron Machines April 8-12, 2013

Bologna, Italy

${ }^{*}$ Speaker. 


\section{Introduction}

During the 2010 and 2011 data-taking periods, the ATLAS [1] and CMS [2] experiments at the Large Hadron Collider (LHC) [3] collected a total of more than $5 \mathrm{fb}^{-1}$ of pp-collisions at $7 \mathrm{TeV}$ center-of-mass energy. The data sample recorded in 2011 has already been exploited to perform many studies of $b$-quark hadron properties, an important part of the physics program for both experiments.

\section{Trigger selection strategies}

Both experiments, even if with some design differences, implement a trigger system comprising a first hardware-based layer, followed by a software-based decision refinement. The selection of $b$-hadron decays presented in the following is based on single or dimuon signatures. Unprescaled single-muon triggers produced a manageable rate until the end of the low luminosity 2010 datataking period. Starting from 2011, for bandwidth reasons, these were mostly replaced by dimuon signatures, with typical muon thresholds set at 4 or $6 \mathrm{GeV}$. In order to cope with the increasing luminosity, these selections were complemented introducing additional requests, such as fitting both muon tracks to a common vertex, applying a mass constraints and, for CMS, requiring a minimum flight length. Figure 1 shows the distribution of the invariant mass of dimuon pairs as selected by ATLAS [4] and CMS [5].
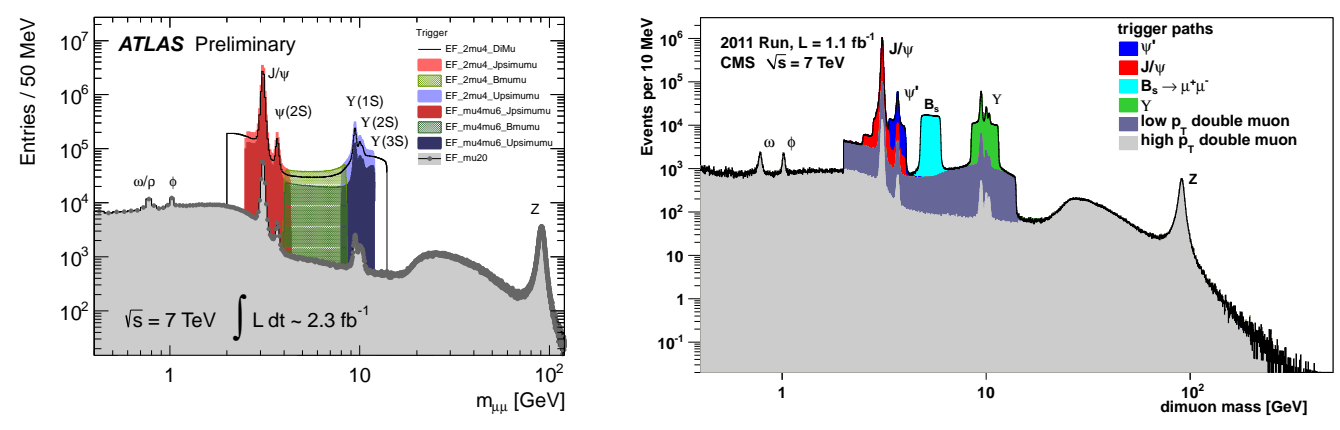

Figure 1: Invariant mass distribution of dimuon pairs selected by $B$-physics triggers in ATLAS (left) [4] and CMS (right) [5], during the 2011 data-taking period. Events selected by dimuon selections and triggers implementing specific mass constraints are shown in different colors and compared to those selected by single muon triggers (gray).

\section{Measurement of inclusive $B$ lifetime and $B_{d}^{0}$ lifetime}

As a first validation of the ingredients needed to reconstruct exclusive decays, ATLAS performed a measurement of the average lifetime of $b$-hadrons in transitions to final states with a $J / \psi$ decaying to two muons [6]. The measurement was done using the $35 \mathrm{pb}^{-1}$ collected in 2010 . In this case the transverse momentum of the $b$-hadron is not known, so it is approximated by the $J / \psi$ transverse momentum. Consequently, a pseudo-proper time is built for the reconstructed $J / \psi$ candidates, instead of the proper time of the decaying $b$-hadron, requiring a Monte Carlo correction factor. The pseudo-proper time is defined as 


$$
t^{*}=\frac{L_{x y} m_{P D G}^{J / \psi}}{p_{T}(J / \psi)}
$$

where $L_{x y}$ and $p_{T}(J / \psi)$ are the projections of the decay length and momentum of the $J / \psi$ on the transverse plane. A simultaneous unbinned maximum likelihood fit is then performed for the mass and pseudo-proper time of the $J / \psi$ candidates. The measured average lifetime is [6]

$$
<\tau_{b}>=1.489 \pm 0.016(\text { stat }) \pm 0.043 \text { (syst) ps }
$$

which is in good agreement with the world average [7] and the CDF measurement [8].

Using the same 2010 data sample, ATLAS started testing the reconstruction of exclusive decays, such as $B_{d}^{0} \rightarrow J / \psi\left(\mu^{+} \mu^{-}\right) K^{* 0}\left(K^{+} \pi^{-}\right)$. This paved the way to $B_{s}^{0}$ measurements, allowing to validate detector resolution and reconstruction techniques on a sample with much larger statistics. Using 2011 data, the same $B_{d}^{0}$ decay was also analyzed in the context of hadron flavor tagging studies.

ATLAS also measured the lifetime of the $B_{d}^{0}$ meson in the decay $B_{d}^{0} \rightarrow J / \psi\left(\mu^{+} \mu^{-}\right) K_{S}^{0}\left(\pi^{+} \pi^{-}\right)$ [11], on a data sample corresponding to $4.9 \mathrm{fb}^{-1}$ collected in 2011. The measured value of the $B_{d}^{0}$ lifetime is [11]

$$
\tau\left(B_{d}^{0}\right)=1.509 \pm 0.012(\text { stat }) \pm 0.018(\text { syst }) \text { ps }
$$

which is consistent with the world average value [7].

\section{Measurement of $B_{s}^{0}$ lifetime}
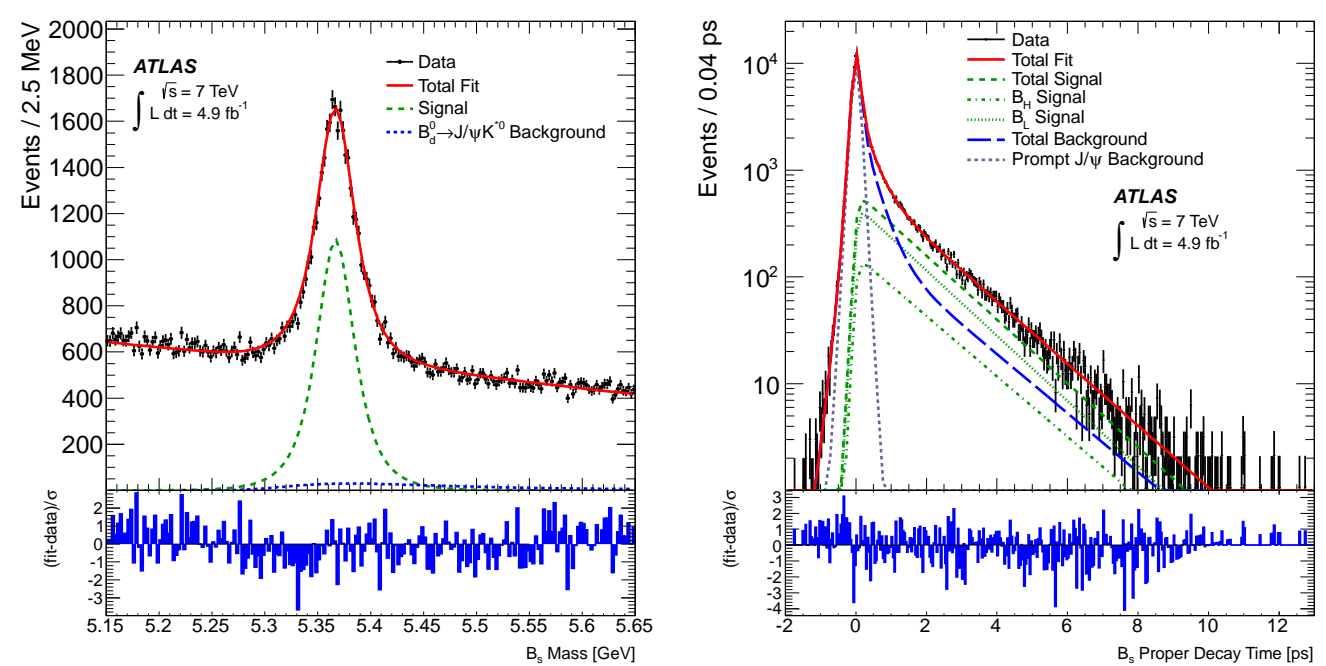

Figure 2: Projection of the fitted probability density function onto the mass axis (left) and the proper decay time (right) for $B_{s}^{0}$ candidates reconstructed by ATLAS in the $B_{s}^{0} \rightarrow J / \psi\left(\mu^{+} \mu^{-}\right) \phi\left(K^{+} K^{-}\right)$channel [9]. The errors displayed for data points are statistical only. 

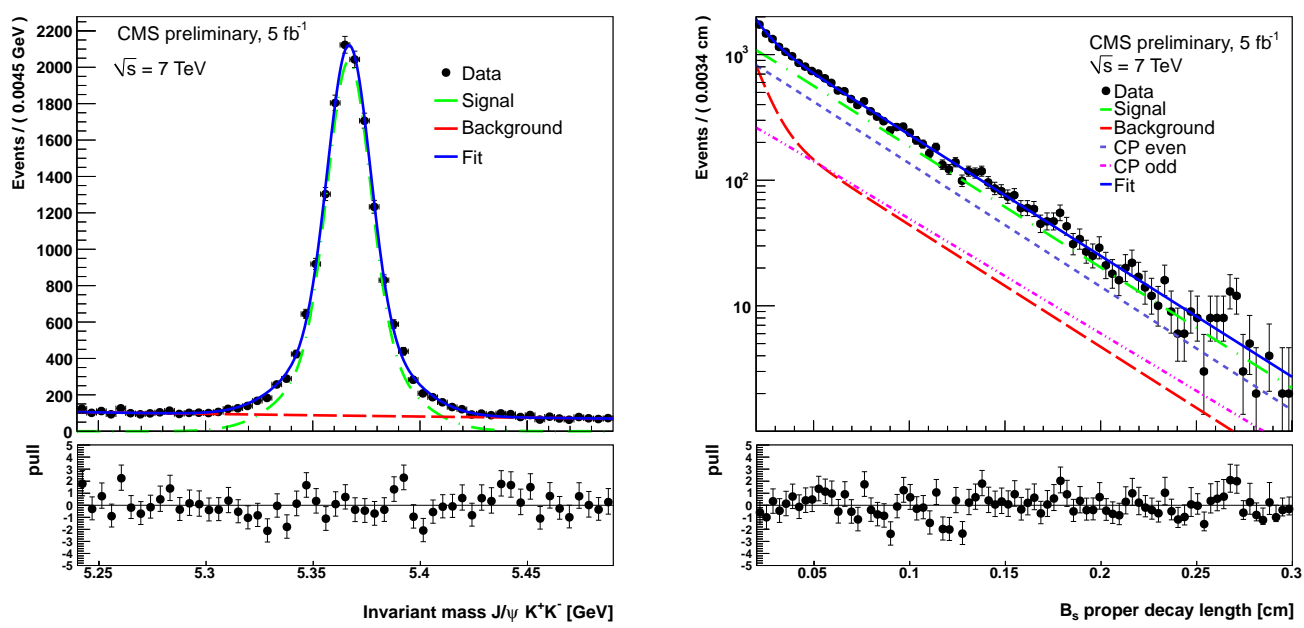

Figure 3: Projection of the fitted probability density function onto the mass axis (left) and the proper decay length (right) for $B_{s}^{0}$ candidates reconstructed by CMS in the $B_{s}^{0} \rightarrow J / \psi\left(\mu^{+} \mu^{-}\right) \phi\left(K^{+} K^{-}\right)$channel [10]. The errors displayed for data points are statistical only.

The lifetime of the $B_{s}^{0}$ meson was measured by both ATLAS [9] and CMS [10] on 2011 data, in the $B_{s}^{0} \rightarrow J / \psi\left(\mu^{+} \mu^{-}\right) \phi\left(K^{+} K^{-}\right)$channel, as part of $C P$-violation studies. The analysis is performed similarly in both experiments, starting from the selection of two opposite-sign muons and two opposite-sign tracks, matching respectively the $J / \psi$ and $\phi$ mass. The dimuon invariant mass is then constrained to the nominal $J / \psi$ mass and the selected track quadruplet is fitted to a common vertex, applying quality criteria on $\chi^{2}$. CMS applies an additional selection requiring a minimum flight length for the reconstructed vertex. Once $B_{s}^{0}$ candidates are selected, a simultaneous unbinned maximum likelihood fit of $B_{s}^{0}$ mass, proper decay time and three decay angles is performed. Among other parameters, the average lifetime between heavy and light states of the $B_{s}^{0}$ meson is extracted. Figures 2 and 3 show respectively the projections of the five-dimensional likelihood adopted for the fit of the mass and proper decay time for ATLAS and of the mass and proper decay length for CMS.

The following measurements were extracted by the two experiments $[9,10]$ :

$$
\begin{gathered}
\Gamma_{s}=0.677 \pm 0.007 \text { (stat) } \pm 0.004 \text { (syst) } \mathrm{ps}^{-1} \quad(\text { ATLAS }) \\
c \tau\left(B_{s}^{0}\right)=0.04580 \pm 0.00059 \text { (stat) } \pm 0.00022(\text { syst }) \quad c m \quad(C M S)
\end{gathered}
$$

The two values are mutually compatible and consistent with the world average value [7].

\section{Measurement of $\Lambda_{b}^{0}$ lifetime}

The lifetime of the $\Lambda_{b}^{0}$ baryon, the lightest one containing a $b$-quark, has been measured by ATLAS [11] and CMS [12] in the decay $\Lambda_{b}^{0} \rightarrow J / \psi\left(\mu^{+} \mu^{-}\right) \Lambda^{0}\left(p+\pi^{-}\right)$. As this chain shares the decay topology and most systematic uncertainties with the $B_{d}^{0} \rightarrow J / \psi K_{S}^{0}$ decay, the lifetime ratio of the $\Lambda_{b}^{0}$ relative to that of the $B_{d}^{0}$ can be measured precisely and compared to theoretical predictions. This is of particular interest, since different values for $R=\tau\left(\Lambda_{b}^{0}\right) / \tau\left(B_{d}^{0}\right)$ are predicted by different calculations $[13,14]$. Furthermore, the measurements performed by Tevatron experiments $[15,16]$ 
show a tension at the level of $2 \sigma$, calling for more experimental results.

Also in this case, the analysis procedure is similar for both experiments; two opposite-sign muons and two opposite-sign tracks are selected, matching respectively the $J / \psi$ and $\Lambda^{0}$ mass. The dimuon invariant mass is then constrained to the nominal $J / \psi$ mass and the four tracks are fitted to a cascade vertex; a minimum flight length is requested for the $\Lambda^{0}$, and quality criteria are applied on vertex $\chi^{2}$. Reconstructed $\Lambda^{0}$ candidates enter a simultaneous unbinned maximum likelihood fit of mass and proper decay time. Figures 4 and 5 show respectively the projections of the fit likelihood onto the mass and proper decay time for ATLAS and CMS.

The following measurements were extracted by the two experiments [11, 12]:

$$
\begin{array}{cc}
\tau\left(\Lambda_{B}^{0}\right)=1.449 \pm 0.036(\text { stat }) \pm 0.017(\text { syst }) \text { ps } & \text { (ATLAS) } \\
\tau\left(\Lambda_{B}^{0}\right)=1.503 \pm 0.052(\text { stat }) \pm 0.031 \text { (syst) ps } & (\text { CMS })
\end{array}
$$

The two values are mutually compatible and fall in between the previous Tevatron measurements. ATLAS also released a determination of the ratio of the $\Lambda_{b}^{0}$ and $B_{d}^{0}$ lifetimes [11]

$$
R=\tau\left(\Lambda_{b}^{0}\right) / \tau\left(B_{d}^{0}\right)=0.960 \pm 0.025 \text { (stat) } \pm 0.016 \text { (syst) }
$$

which is compatible with next-to-leading order theoretical predictions, seeming to favor the ones based on Heavy Quark Expansion [13].
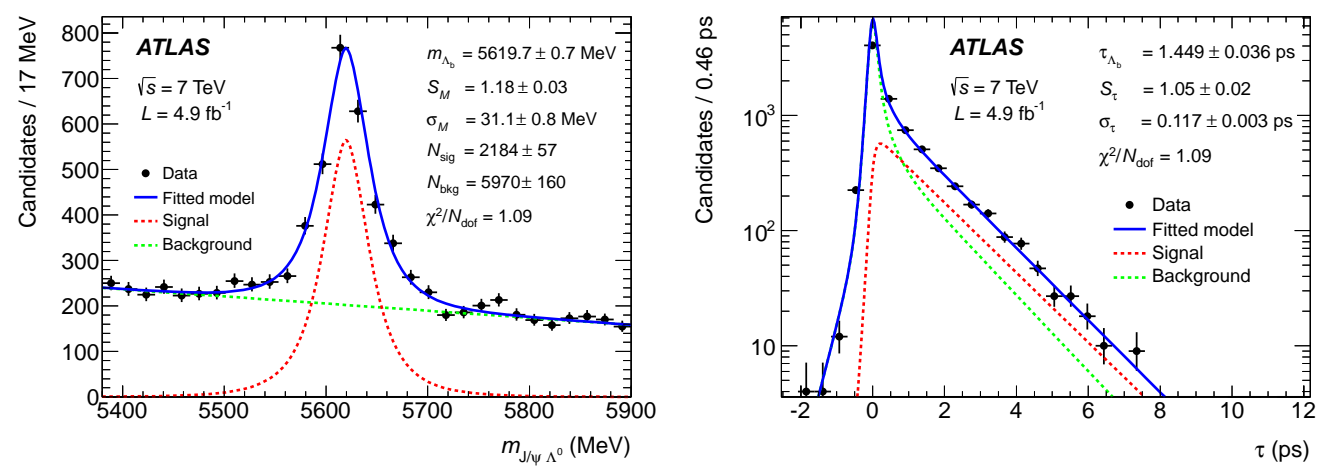

Figure 4: Projection of the fitted probability density function onto the mass axis (left) and the proper decay time (right) for $\Lambda_{b}^{0}$ candidates reconstructed by ATLAS in the $\Lambda_{b}^{0} \rightarrow J / \psi\left(\mu^{+} \mu^{-}\right) \Lambda^{0}\left(p+\pi^{-}\right)$channel [11]. The errors displayed for data points are statistical only.

\section{Conclusions}

The measurements of lifetimes of the $B_{d}^{0}$ and $B_{s}^{0}$ mesons and of the $\Lambda_{b}^{0}$ baryon, performed using data collected by the ATLAS and CMS experiments during 2011, have been presented. The results of the two experiments are compatible among them and in good agreement with previous measurements, at the same time demonstrating excellent detector performance and analysis accuracy. 

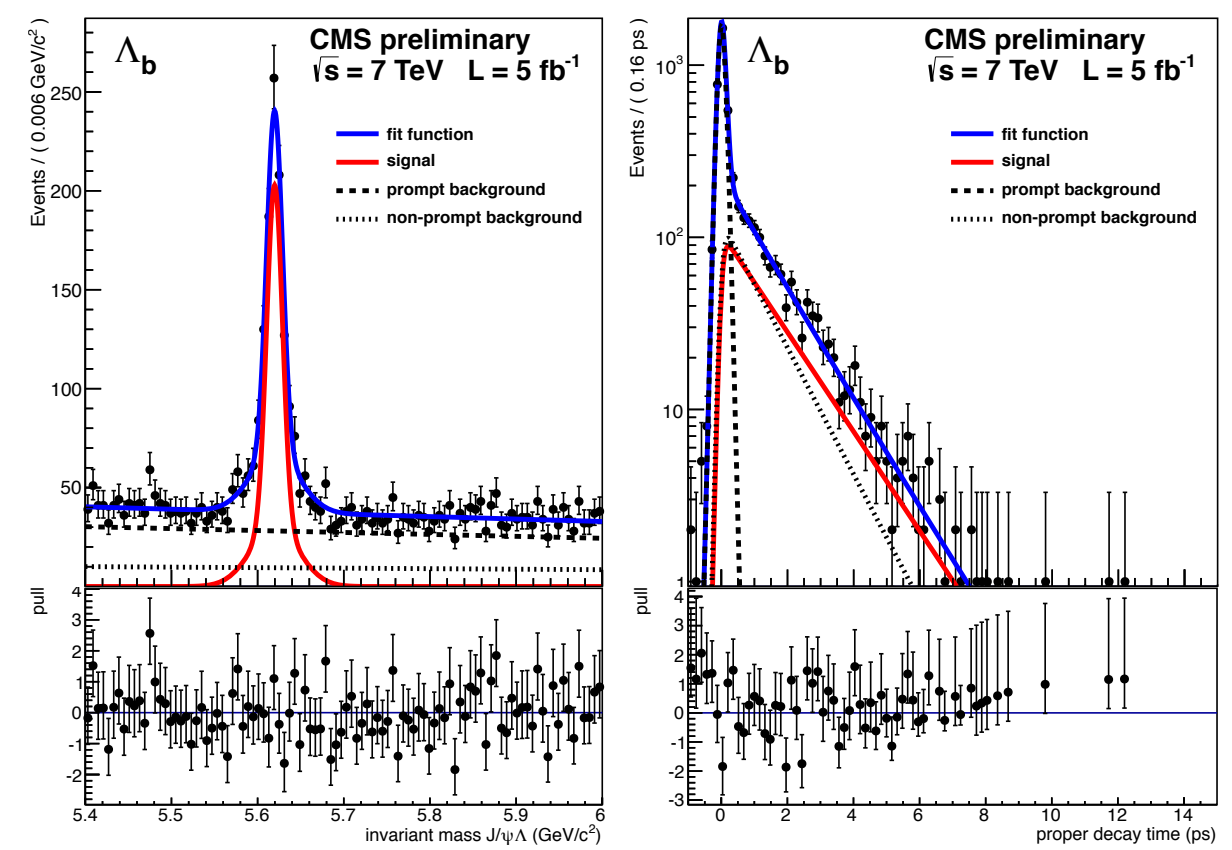

Figure 5: Projection of the fitted probability density function onto the mass axis (left) and the proper decay time (right) for $\Lambda_{b}^{0}$ candidates reconstructed by CMS in the $\Lambda_{b}^{0} \rightarrow J / \psi\left(\mu^{+} \mu^{-}\right) \Lambda^{0}\left(p+\pi^{-}\right)$channel [12]. The errors displayed for data points are statistical only.

\section{References}

[1] ATLAS Collaboration, The ATLAS Experiment at the CERN Large Hadron Collider, 2008 JINST 3 S08003.

[2] CMS Collaboration, The CMS Experiment at the CERN Large Hadron Collider, 2008 JINST 3 S08004.

[3] L. Evans and Ph. Bryant, editors, LHC Machine, 2008 JINST 3 S08001.

[4] ATLAS Collaboration, B-physics public results, available at http://twiki.cern.ch/twiki/bin/view/AtlasPublic/BPhysPublicResults.

[5] CMS Collaboration, B-physics and quarkonia public results, available at http://twiki.cern.ch/twiki/bin/view/CMSPublic/PhysicsResultsBPH.

[6] ATLAS Collaboration, Measurement of the average B lifetime in inclusive $B \rightarrow J / \psi X \rightarrow \mu^{+} \mu^{-} X$ decays with the ATLAS detector, ATLAS-CONF-2011-145, http://cdsweb.cern.ch/record/1389455.

[7] J. Beringer, et al., The Review of Particle Physics, Phys. Rev. D 86 (2012) 010001.

[8] CDF Collaboration, Measurement of B hadron lifetimes using $J / \psi$ final states at CDF, Phys. Rev. D 57, 5382-5401 (1998).

[9] ATLAS Collaboration, Time-dependent angular analysis of the decay $B_{s}^{0} \rightarrow J / \psi \phi$ and extraction of $\Delta \Gamma_{s}$ and the CP-violating weak phase $\phi_{s}$ by ATLAS, JHEP 12 (2012) 072.

[10] CMS Collaboration, Measurement of the $B_{s}$ lifetime difference, CMS-BPH-11-006, http://cds.cern.ch/record/1484686. 
[11] ATLAS Collaboration, Measurement of the $\Lambda_{b}^{0}$ lifetime and mass in the ATLAS experiment, Phys. Rev. D 87 (2013) 032002.

[12] CMS Collaboration, Measurement of the $\Lambda_{b}^{0}$ lifetime in pp collisions at $\sqrt{s}=7 \mathrm{TeV}$, CMS-BPH-11-013, http: / / cds . cern. ch/record/1544427.

[13] N. Uraltsev, Phys. Lett. B 376 (1996) 303; I. Bigi, M. Shifman, and N. Uraltsev, Ann. Rev. of Nucl. Part. Science 47, 591 (1997); D. Pirjol and N. Uraltsev, Phys. Rev. D 59, 034012 (1999); M. B. Voloshin, Phys. Rev. D 61, 074026 (2000).

[14] C. Tarantino, Eur. Phys. J. C33, S895 (2004); F. Gabbiani, A. I. Onishchenko, and A. A. Petrov, Phys. Rev. D 70, 094031(2004).

[15] CDF Collaboration, Measurement of b hadron lifetimes in exclusive decays containing a $J / \psi$ in $p \bar{p}$ collisions at $\sqrt{s}=1.96$ TeV, Phys. Rev. Lett. 106 (2011) 121804.

[16] D0 Collaboration, Measurement of the $\Lambda_{b}^{0}$ lifetime in the exclusive decay $\Lambda_{b}^{0} \rightarrow J / \psi \Lambda^{0}$ in $p \bar{p}$ collisions at $\sqrt{s}=1.96$ TeV, Phys. Rev. D 85 (2012) 112003. 